\title{
PURIFICAÇÃO DE BIODIESEL POR ADSORÇÃO EM CARVÃO ATIVADO
}

\author{
F. H. O.SILVA ${ }^{1}$, M. F. VIEIRA ${ }^{2}$, N.C. PEREIRA ${ }^{3}$ e D. R. AGUIAR ${ }^{4}$ \\ 1,2,3,4 Universidade Estadual de Maringá, Departamento de Engenharia Química \\ E-mail para contato: fabiooliveira.eq@gmail.com
}

\begin{abstract}
RESUMO - Neste trabalho foi avaliada a purificação de biodiesel por adsorção em carvão ativado de ossos bovinos modificado com ácido nítrico. O biodiesel foi produzido a $30^{\circ} \mathrm{C}$ por transesterificação etílica de óleo de soja degomado catalisada por hidróxido de sódio. $\mathrm{O}$ excesso de etanol foi retirado por evaporação e a glicerina bruta por decantação. O biodiesel resultante foi purificado por adsorção com carvão ativado e por lavagem úmida. As soluções obtidas foram caracterizadas para a comparação dos dois métodos de purificação, tendo apresentado resultados semelhantes. Foi realizado um estudo sobre a cinética de adsorção de glicerol em solução de glicerina PA em água destilada, e glicerol no biodiesel produzido em laboratório. Os modelos cinéticos de pseudo-primeira ordem e pseudo-segunda ordem de Lagergren foram os que melhor se ajustaram para a solução de glicerina em água e para a solução de glicerol no biodiesel respectivamente. Por meio de ensaios de equilíbrio obtêve-se a isoterma de adsorção para glicerina em água e o modelo linear apresentou o melhor ajuste aos dados, na faixa de concentração testada.
\end{abstract}

\section{INTRODUÇÃO}

O aumento da demanda global por energia tem impulsionado pesquisas sobre fontes alternativas e renováveis de energia. Um exemplo de combustível renovável é o biodiesel, que é produzido a partir de triacilgliceróis de origem vegetal e cujo subproduto principal é o glicerol, substância essa que tem pouco valor combustível Peereboon et al. (2006).

As especificações do biodiesel como produto final, no Brasil, são determinadas pela Agencia Nacional do Petróleo, Gás Natural e Biocombustíveis (ANP). Das diversas propriedades que devem ser contoroladas para atingir essas especificações, uma das principais é a quantidade de glicerol livre, pois essa está diretamente relacionada com a efetividade do processo de purificação ANP (2008). Um elevado teor de glicerol no biodiesel pode causar problemas. Durante o armazenamento, devido a sua separação, e durante o uso, pela formação de de depósitos nos bicos injetores dos motores e por promover o aumento das emissões de aldeidos Mittelbach (1996).

A técnica mais comum para a remoção de impurezas do biodiesel, tais como glicerol, resíduos de catalisadores, sais, álcool residual, entre outros é a lavagem úmida. Devido a grande quantidade de efluentes produzidos nesse processo, novas metodologias de purificação têm sido propostas, dentre as quais se destaca a lavagem a seco, ou adsorção Faccini et al. (2011). 
Nesse contexto os objetivos deste trabalho foram: produzir biodiesel a partir de óleo de soja degomado, realizar sua purificação por adsorção utilizando carvão ativado de ossos bovinos modificado com ácido nítrico e por lavagem úmida, caracterizar o biodiesel purificado e comparar os resultados entre os dois métodos de purificação, analizar as cinéticas de adsorção de glicerol em biodiesel e em solução aquosa e determinar a isoterma de adsorção de glicerol em solução aquosa.

\section{MATERIAIS E MÉTODOS}

Nos experimentos foram utilizados, óleo de soja degomado fornecido pela cooperativa agroindustrial Cocamar de Maringá/PR, etanol anidro (99,8 \% INPM), fornecido pela destilaria Renuka de São Pedro do Ivaí/PR, carvão ativado de ossos bovinos, fornecido pela Bonechar de Maringá/PR, hidróxido de sódio em micro pérolas, da marca Fmaia e ácido nítrico 65 \%, da marca Fmaia.

Todas as análises de teor de glicerol livre no biodiesel e em solução aquosa foram realizadas por meio de uma metodologia modificada, baseada no método oficial da AOCS para análise de glicerol livre em óleos e gorduras (Ca 14-56) conforme Dantas (2006).

Os ésteres foram obtidos por reação de transesterificação etílica de óleo de soja degomado a $30{ }^{\circ} \mathrm{C}$, catalizada por hidróxido de sódio, de acordo com procedimento descrito por Gomes et al. (2011). Ao final da reação, a mistura reacional foi submetida a um evaporador rotativo sob vácuo de $600 \mathrm{mmHg}$ e temperatura em torno de $65^{\circ} \mathrm{C}$ para a recuperação do etanol em excesso. Após a evaporação do etanol, a mistura foi colocada em um funil de separação e deixada em repouso para a decantação da glicerina bruta e posterior separação das fases.

Para a modificação química da superfície do adsorvente foram adicionados $5 \mathrm{~g}$ de carvão ativado em um béquer de $50 \mathrm{~mL}$ com ácido nítrico a $65 \%$ e a mistura foi agitada por uma hora a $60{ }^{\circ} \mathrm{C}$. Em seguida, o ácido nítrico foi removido e a amostra de carvão modificado foi lavada com água destilada e colocada em estufa a $110^{\circ} \mathrm{C}$ de acordo com Abdel-Nasser e El-Hendawy (2003). Posteriormente foram realizados ensaios de adsorção com carvões tratados de acordo com a metodologia descrita acima, utilizando diferentes soluções de ácido nítrico $(1 \%, 2 \%, 3 \%, 4 \%$ e $65 \%$ ) e por apresentar bons resultados de remoção de glicerol, foi escolhido o tratamento com a solução ácida de menor concentração, $(1 \%)$ visando à redução de custos. A área superficial, o volume total dos poros e o diâmetro médio dos poros dos carvões, modificado (CAM) e não modificado (CA), foram determinados por adsorção de nitrogênio através do método BET a 77,4 $\mathrm{K}$ sobre uma faixa de pressão relativa, $\mathrm{P} / \mathrm{P}_{0}$, variando de 0,0 a 1,0 com o equipamento Nova 1200 Series da QuantaChrome. Os valores obtidos são apresentados na Tabela 1.

Tabela 1 - Caracterização física dos carvões ativado (CA) e ativado modificado (CAM)

\begin{tabular}{|c|c|c|}
\hline Parâmetros & CA & CAM \\
\hline Diâmetro médio $(\mathrm{mm})$ & 0,595 & --- \\
\hline Área superficial BET $\left(\mathrm{m}^{2} \cdot \mathrm{g}^{-1}\right)$ & 104,3 & 235,6 \\
\hline Diâmetro dos poros $(\AA)$ & 30,56 & 27,19 \\
\hline Volume dos microporos $\left(\mathrm{cm}^{3} \cdot \mathrm{g}^{-1}\right)$ & 0,051 & 0,116 \\
\hline Área de microporos $\left(\mathrm{m}^{2} \cdot \mathrm{g}^{-1}\right)$ & 144,2 & 326,4 \\
\hline
\end{tabular}

Todos os ensaios de adsorção foram realizados em uma incubadora Shaker da marca 
MARCONI, modelo MA 420, a $25^{\circ} \mathrm{C}$ e sob agitação de 80 RPM. Na purificação por adsorção, do biodiesel a ser caracterizado, foram adicionados $20 \pm 0,0001 \mathrm{~g}$ de biodiesel e $1 \% \mathrm{~m} / \mathrm{m}$ de carvão ativado em Erlenmeyers de $125 \mathrm{~mL}$. O carvão foi mantido em contato com o biodiesel por $180 \pm 1$ min e separado por por filtração simples ao final desse período. A purificação por lavagem úmida, por sua vez, foi realizada de acordo com a metodologia descrita por Faccini et al. (2011).

Para a caracterização do biodiesel purificado por adsorção e por lavagem úmida foram determinados, o teor de glicerol livre, a massa específica em um densímetro da marca Anton Paar, modelo DMA 5000, a viscosidade cinemática a $40{ }^{\circ} \mathrm{C}$ em reômetro digital Brookfield modelo DVIII, o índice de acidez conforme o método oficial da AOAC - 940-28, o teor de umidade pelo método de Karl Fischer em um equipamento Karl Fischer da marca Analyser modelo Umidade Controle KF-1000, o índice de saponificação de acordo com a norma AOCS Cd 3-52 e o teor de ésteres por cromatografia em fase gasosa utilizado-se um cromatógrafo da marca Varian, modelo CP 3800, com detector de ionização de chama (DIC), contendo uma coluna capilar especifica para separação de ésteres (BP - X70 - SGE) de $30 \mathrm{~m}$ x 0,25 mm.

Os ensaios cinéticos foram realizados conforme o mesmo procedimento, tanto para a solução de glicerina PA em água, denominada Solução 1, quanto para a solução de glicerol residual em biodiesel (que corresponde ao biodiesel produzido em laboratório), denominada solução 2. Esse consistiu na preparação de oito amostras em duplicata, sendo cada uma delas preparada pela adição de $20 \pm 0,0001 \mathrm{~g}$ de solução e $5 \%$ em massa de adsorvente a erlenmeyers de $125 \mathrm{ml}$. Os recipientes foram mantidos a $25{ }^{\circ} \mathrm{C}$ sob agitação de 80 RPM por tempos predeterminados de 10, 15, 30, 45, 60, 90, 120 e 180 minutos, variando de acordo com a amostra. Ao final do tempo de adsorção de cada amostra o adsorvente foi retirado por filtração simples e o teor de glicerol residual foi determinado. Os dados experimentais de cinética foram comparados com os modelos cinéticos de pseudo-primeira ordem e pseudo-segunda ordem de Lagergren, cujas equações de ajuste são mostradas nas equações (1) e (2) respectivamente Lagergren (1898).

$$
\begin{aligned}
& q t=q e q-q e q * \exp ^{(-k 1 * t)} \\
& q t=\left(k 2 *\left(q e q^{2}\right) * t\right) /(1+(k 2 * q e q * t))
\end{aligned}
$$

Para determinação da isoterma de adsorção de glicerol em solução aquosa preparou-se uma solução de glicerina PA em água de concentração 2,717 (mg / g de solução), (denominada solução 1:1) com a qual foram preparadas amostras por meio de 9 diluições em duplicata com água destilada, totalizando $20 \pm 0,0001 \mathrm{~g}$ cada amostra. Foram adicionadas alíquotas de $(20 ; 18 ; 16 ; 14$; $12 ; 10 ; 8 ; 6$ e 4) $\pm 0,0001$ gramas da solução $1: 1$ às amostras de números 1 a 9 respectivamente. Às amostras obtidas a partir desses procedimentos foram adicionados 7,5\% em massa de adsorvente que foi mantido em contato com a solução por $180 \pm 1$ minuto, a $25{ }^{\circ} \mathrm{C}$ e sob agitação de 80 RPM. Passado esse tempo o carvão foi separado da solução por filtração simples. Na etapa de determinação do teor de glicerol a concentração da solução titulante foi diluída em 4 vezes em relação ao estabelecido pela metodologia AOCS (Ca 14-56). As isotermas de Languimuir, Freundlish e Linear, (Peerebon et al. 2006 e McCabe 1993) que são apresentadas nas equações (3), (4) e (5) respectivamente, foram ajustadas aos dados de equilíbrio obtidos.

$$
\begin{aligned}
& q e q=(K f * C e q)^{\left(\frac{1}{n}\right)} \\
& q e q=(q \max * K L * C e q) /(1+K L * C e q) \\
& q e q=A * C e q
\end{aligned}
$$




\section{RESULTADOS E DISCUSSÃO}

\subsection{Comparação dos Métodos de Purificação}

Tabela 2 - Caracterização físico-química do biodiesel purificado por adsorção e por lavagem

\begin{tabular}{|c|c|c|c|}
\hline Método de Purificação & Limite ANP & Adsorção & Lavagem Úmida \\
\hline Propriedade & & & \\
\hline Massa específica, $25{ }^{0} \mathrm{C}\left(\mathrm{kg} / \mathrm{m}^{3}\right)$ & $0,850-0,900$ & 0,874 & 0.872118 \\
\hline Viscosidade cinemática, $40{ }^{\circ} \mathrm{C}\left(\mathrm{mm}^{2} / \mathrm{s}\right)$ & $3,0-6,0$ & 3,42 & 4,07 \\
\hline Acidez $(\mathrm{mg} \mathrm{KOH} / \mathrm{g})$ & 0,5 máximo & $0,4444 \pm 0,016$ & $1,4496 \pm 0,016$ \\
\hline Teor de umidade $(\%)$ & 0,5 máximo & $0,318 \pm 0,004$ & $0,0634 \pm 0,004$ \\
\hline Glicerol $(\%)$ & 0,02 máximo & $0,0013 \pm 0,0002$ & $0,0056 \pm 0,0002$ \\
\hline Teor de Ésteres $(\%)$ & 96,5 mínimo & $94,95 \pm 1,13$ & $93,20 \pm 9,70$ \\
\hline
\end{tabular}

Como pode ser visto na Tabela 2 o teor de ésteres obtido pelo biodiesel purificado por adsorção foi superior àquele obtido por lavagem úmida, mas ainda ligeiramente abaixo das normas da ANP. Este resultado mostra a necessidade de aprimoramento do método de adsorção, porém os resultados obtidos parecem promissores. Todos os outros parâmetros analizados apresentaram valores dentro das especificações da ANP para o biodiesel purificado por adsorção, entretanto, para o biodiesel purificado por lavagem, o índice de acidez encontra-se acima do permitido, o que demonstra a potencialidade do processo de adsorção como método de purificação do biodiesel.

\subsection{Cinéticas de Adsorção}

As Figuras 1 e 2 apresentam as cinéticas de adsorção de glicerol a $25^{\circ} \mathrm{C}$ para as soluções de glicerina PA em água destilada (solução 1) e glicerol residual no biodiesel (solução 2) respectivamente.

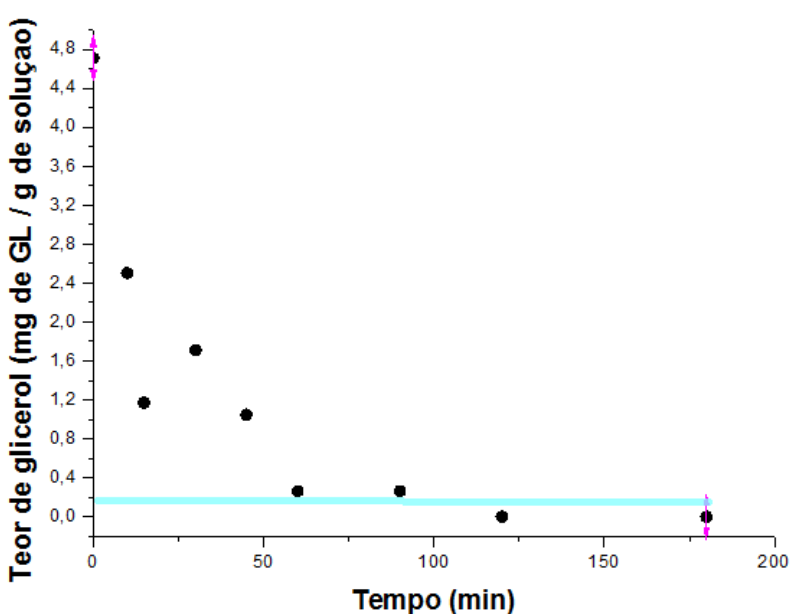

Figura 1 - Cinética de adsorção de glicerol para a solução 1 à $25^{\circ} \mathrm{C}$.

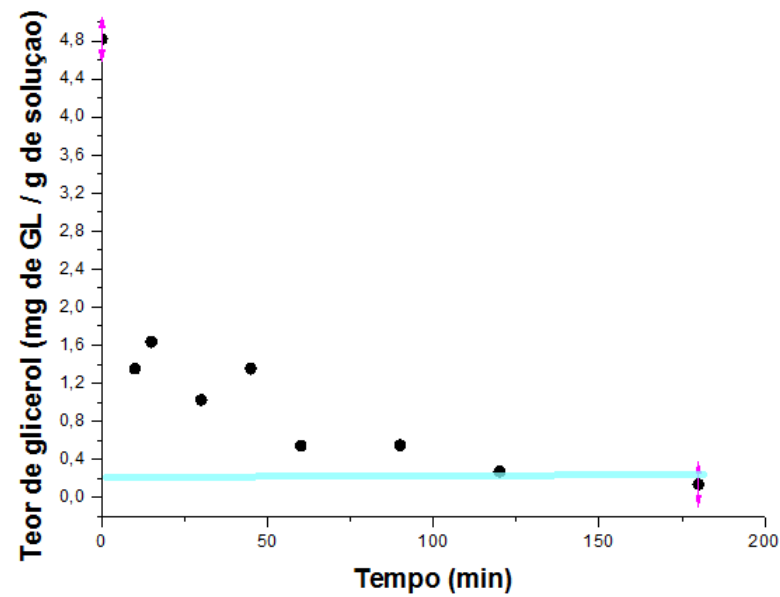

Figura 2 - Cinética de adsorção de glicerol para a solução 2 à $25^{\circ} \mathrm{C}$. 
Comparando-se as Figuras 1 e 2 verifica-se que, o equilíbrio foi estabelecido por volta de 150 minutos com teor de glicerol aproximadamente nulo para ambas as soluções. Verifica-se também que o valor máximo permitido pela ANP, 0,2 (mg de GL / g de solução) que está indicado pelas retas horizontais em azul, foi alcançado em aproximadamente 120 minutos para as soluções 1 e 2, considerando as amplitudes dos erros associados às medidas de GL. Esses resultados demonstram que o tempo necessário para que o sistema entre em equilíbrio, assim como a concentração de glicerol no equilíbrio para ambas as soluções estudadas não é afetado por outros contaminantes presentes no biodiesel, ou pelo próprio biodiesel.

O Quadro 1 apresenta os parâmetros dos modelos cinéticos de pseudo-primeira ordem e pseudo-segunda ordem de Lagergrem, seus valores, erros e os coeficientes de correlação dos ajustes aos dados cinéticos das soluções 1 e 2 .

Quadro 1- Parâmetros cinéticos de ajuste dos modelos de pseudo-primeira ordem e pseudo segunda ordem de Lagergren aos dados de cinética de adsorção para as soluções 1 e 2.

\begin{tabular}{|c|c|c|c|c|c|}
\hline & Modelo & $\overline{\mathrm{R}^{2}}$ & Parâmetros & Valor & Erro \\
\hline \multirow{4}{*}{ Solução 1} & \multirow[t]{2}{*}{ Pseudo-primeira ordem } & \multirow[t]{2}{*}{0,97466} & $\mathrm{k}_{1}\left(\min ^{-1}\right)$ & 0,03413 & 0,0046 \\
\hline & & & $\mathrm{q}_{\mathrm{eq}}(\mathrm{mg} / \mathrm{g})$ & 0,40824 & 0,0157 \\
\hline & \multirow[t]{2}{*}{ Pseudo-segunda ordem } & \multirow[t]{2}{*}{0,97598} & $\mathrm{k}_{2}\left(\mathrm{~g} \cdot \mathrm{mg}^{-1} \cdot \mathrm{min}^{-1}\right)$ & 0,08885 & 0,0226 \\
\hline & & & $\mathrm{q}_{\mathrm{eq}}(\mathrm{mg} / \mathrm{g})$ & 0,47801 & 0,0263 \\
\hline \multirow{4}{*}{ Solução 2} & \multirow[t]{2}{*}{ Pseudo-primeira ordem } & \multirow[t]{2}{*}{0,95814} & $\mathrm{k}_{1}\left(\min ^{-1}\right)$ & 0,08682 & 0,0172 \\
\hline & & & $\mathrm{q}_{\mathrm{eq}}(\mathrm{mg} / \mathrm{g})$ & 0,37571 & 0,0126 \\
\hline & \multirow[t]{2}{*}{ Pseudo-segunda ordem } & \multirow[t]{2}{*}{0,97598} & $\mathrm{k}_{2}$ (g.mg-1.min-1) & 0,30141 & 0,0480 \\
\hline & & & $\mathrm{q}_{\mathrm{eq}}(\mathrm{mg} / \mathrm{g})$ & 0,41442 & 0,0092 \\
\hline
\end{tabular}

A partir do Quadro 1, ao considerar a solução 1, verifica-se o valor de $\mathrm{R}^{2}$ do ajuste ao modelo de pseudo-segunda ordem é ligeiramente superior ao correspodente valor do modelo de pseudo-primeira ordem. No entanto, os erros dos parâmetros $\mathrm{k}_{2}$ e $\mathrm{q}_{\mathrm{eq}}$ daquele modelo são consideravelmente maiores do que os erros dos valores $\mathrm{k}_{1}$ e $\mathrm{q}_{\mathrm{eq}}$ desse modelo, o que indica que o modelo de pseudo-primeira ordem representa melhor os dados cinéticos para a solução 1. Por sua vez, ao considerar-se a solução 2 , verifica-se que para o modelo de pseudo-segunda ordem, tanto seu o valor de $\mathrm{R}^{2}$ é maior, quanto o erro do seu parâmetro qeq é menor quando comparado ao qeq correspondente ao modelo de pseudo-primeira ordem. Isso indica que a cinética de adsorção da solução 2 é melhor representada pelo modelo de pseudo-segunda ordem, o que está de acordo com o resultado obtido por Vasques (2010).

A cinética do processo de adsorção sólido-líquido depende da velocidade relativa entre as etapas do processo, a saber; transporte do adsorvato no seio da solução, transporte por difusão através da camada limite, transporte entre os poros da partícula e a ligação entre o adsorvato e a partícula. As duas primeiras etapas são responsáveis pelo transporte do adsorvato até a superfície do adsorvente e as duas últimas etapas pela adsorção propriamente dita Weber e Smith (1986). Conforme pode ser observado nas Figuras 1 e 2, embora o tempo de equilíbrio e a concentração de equilíbrio de glicerol sejam aproximadamente iguais para ambas as soluções, as etapas iniciais de adsorção foram mais lentas para a solução 2, o que impactou diretamente nos ajustes dos modelos teóricos, de forma que as soluções se ajustaram a modelos cinéticos diferentes. Isso se deve ao fato de que a velocidade de adsorção para a solução 1 no início do processo adsortivo foi maior 
em relação à solução 2 nesta mesma etapa do processo, o que pode ser explicado pela maior resistência ao transporte do glicerol do seio da solução de biodiesel até a camada limite e através da camada limite, conferida principalmente pela presença das grandes moléculas dos ésteres etílicos que são o principal componente da solução 2 .

\subsection{Isoterma de Adsorção}

A Figura 3 apresenta os dados de equilíbrio de adsorção para a solução de glicerol em água e os ajustes das isotermas de adsorção de Languimuir, Freundlich, e Linear. Os valores dos parâmetros de ajuste podem ser encontrados no Quadro 2.

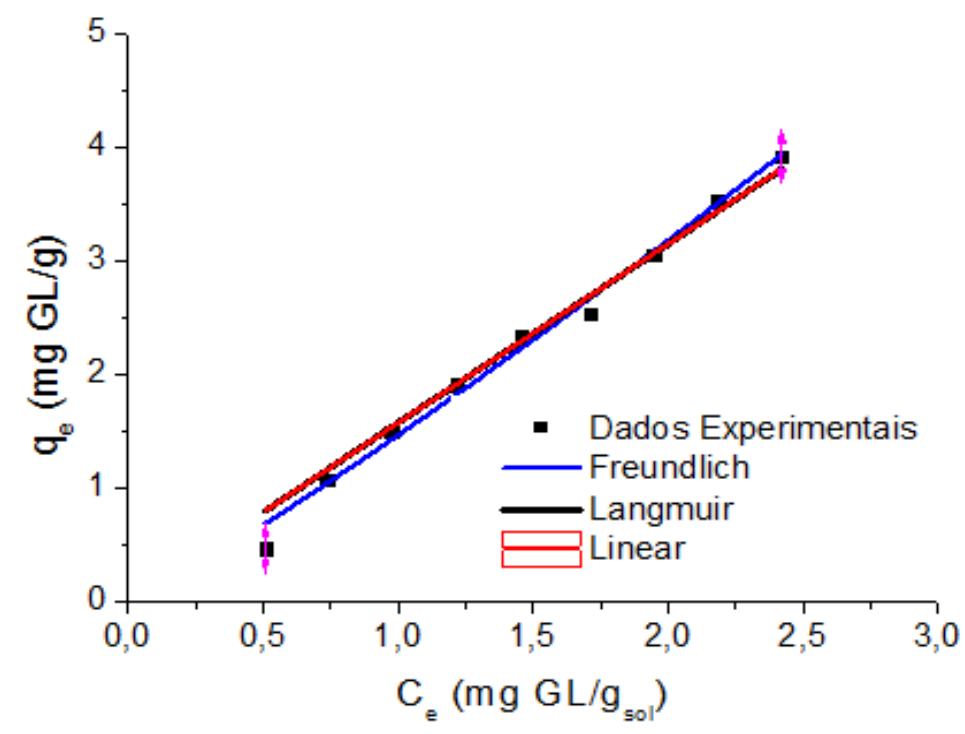

Figura 3: Ajuste dos modelos de Langmuir, Freundlich e linear aos dados de equilíbrio para a solução de glicerol em água.

Quadro 2 - Parâmetros de equilíbrio de adsorção da solução 1.

\begin{tabular}{|c|c|c|c|c|}
\hline Modelo & $\mathrm{R}^{2}$ & Parâmetros & Valor & Erro \\
\hline \multirow{2}{*}{ Freundlich } & 0,990 & $\mathrm{n}(\mathrm{admensional})$ & 0,90 & 0,042 \\
\cline { 3 - 5 } & & $\mathrm{Kf}(\mathrm{g} / \mathrm{g})$ & 1,47 & 0,052 \\
\hline \multirow{2}{*}{ Langmuir } & \multirow{2}{*}{0,982} & $\mathrm{qmax}(\mathrm{mg} / \mathrm{g})$ & 6422,96 & $1,11 \times 10^{6}$ \\
\cline { 3 - 5 } & & $\mathrm{KL}(\mathrm{g} / \mathrm{mg})$ & $2,45 \times 10^{-4}$ & 0,042 \\
\hline Linear & 0,984 & $\mathrm{~A}(\mathrm{~g} / \mathrm{g})$ & 1,58 & 0,027 \\
\hline
\end{tabular}

Peereboon et al. (2006) estudaram a adsorção de glicerol em solução aquosa a $25{ }^{\circ} \mathrm{C}$ com dois tipos distintos de carvão ativado comerciais, ROX e 3310 carbon, numa faixa de concentração de 0,9 a 184,0 mg de glicerol por grama de solução e obtiveram o melhor ajuste para o modelo de Langmuir em toda a faixa de concentração estudada, utilizando para a obtenção dos dados de equilíbrio a cromatografia líquida de alta eficiência. Os erros apresentados pelos parâmetros desse modelo no presente trabalho foram muito elevados, bem como, seu $\mathrm{R}^{2}$ foi o menor dentre os modelos o que demostra não ser esse adequado para a representação dos dados. 
Ao analisar-se os valores de $\mathrm{R}^{2}$ para os modelos de Freundlish e Linear, bem como os erros apresentados por seus parâmetros verifica-se que ambos os modelos são adequados para representar os dados de equilíbrio de adsorção. Ao considerar no entanto, a pequena faixa de concentração estudada, que foi de 0,27 a $2,72 \mathrm{mg}$ de glicerol por grama de solução, verifica-se que que a isoterma de Freundlish, nessas condições, tende a apresentar comportamento linear, o que justifica o fato desse modelo ter apresentado um bom ajuste. Entretanto, o modelo mais adequado para representar os dados de equilíbrio em estudo é o modelo Linear partindo da origem, visto que esse é o modelo que representa a lei de Henry, lei essa que rege o comportamento de soluções diluidas. Por esse motivo, é possível que outros modelos como o de Langmuir, apresentado por Peereboon et al. (2006) se ajustem melhor para uma faixa de concentração de glicerol mais ampla.

\section{CONCLUSÕES}

As propriedades físico-químicas do biodiesel purificado por adsorção, avaliadas por sua caracterização, apresentaram bons resultados quando comparados ao biodiesel purificado por lavagem, o que demonstra o potencial do método proposto.

O tempo de equilíbrio e a concentração final de glicerol em solução, no equilíbrio, foram aproximadamente iguais para as soluções 1 e 2 o que demonstrou que essas variáveis não são influenciados por contaminantes presentes no biodiesel ou pelo próprio biodiesel, entretanto, as curvas de cinética de adsorção das soluções 1 e 2 se ajustaram a modelos teóricos diferentes. Isso se deve a presença das grandes moléculas de ésteres etílicos que compõem a solução 2, que são capazes de diminuir a velocidade das etapas iniciáis do processo adsortivo por meio do aumento da resistência ao transporte do glicerol até a camada limite e através da camada limite.

A isoterma de adsorção para a solução 1, na faixa de concentração estudada, segue o modelo linear, com o coeficiente de correlação igual a 0,984 , o que é condizente com o fato de ter sido utilizada uma solução diluída.

\section{NOMENCLATURA}

GL - Glicerol livre.

$A$ - Parâmetro de ajuste da isoterma linear dado em $\mathrm{g}$ de solução/g de adsorvente.

KL - Constante de equilíbrio de adsorção de Langmuir dada em g/mg.

$C_{e q}$ - Concentração de glicerol em solução no equilíbrio dada em mg/g solução.

$n$ - Expoente na equação da isoterma de Freundlish.

$q_{e q}$ - Massa de adsorvato por unidade de massa de adsorvente no equilíbrio em $\mathrm{mg} / \mathrm{g}$.

$q_{t}$ - Massa de adsorvato por unidade de massa de adsorvente no equilíbrio em $\mathrm{mg} / \mathrm{g}$ em função do tempo.

qmax - Capacidade máxima de adsorção por unidade de massa de adsorvente em (mg/g).

$t$-É o tempo de contato entre o adsorvente e a solução dado em min.

$K f$ - Constante de Freundlish dada em g de solução/g de adsorvente.

$k 1$ - Constante do modelo cinético de adsorção de primeira ordem dada em min $^{-1}$. 
k2 - Constante do modelo cinético de adsorção de segunda ordem dada em (g.mg-1.min-1).

\section{REFERÊNCIAS}

ABDEL-NASSER A.; EL-HENDAWY. Influence of HNO3 oxidation on the structure and adsorptive properties of corncob-based activated carbon. Carbon, v. 41, p. 713 - 722, 2003.

ANP - Agência Nacional do Petróleo, Gás Natural e Biocombustíveis, RESOLUÇÃO ANP № 7, DE 19.3.2008 - DOU 20.3.2008. Disponível em: <www.anp.gov.br>, acessado em 15/09/2013.

A.O.A.C. ASSOCIATION OF OFFICIAL ANALYTICAL CHEMISTS. Official Methods of Analysis of AOAC International. Food composition, additives, natural contaminants. 16. ed. v. 2. Gaithersburg: AOAC, 1997.

AOCS. ASSOCIATION OF OFFICIAL ANALYTICAL CHEMISTS. Official Methods and Recommended Practices of the American Oil Chemists' Society. Champaing, Ill.: AOCS 2004.

FACCINI C. S.; DA CUNHA M. E.; MORAES M. S. A.; KRAUSE L. C.; MANIQUE C. M.; RODRIGUES M. R. A.; BENVENUTTI E. V.; CARAMÃO E. B. Dry Washing in Biodiesel Purification: a Comparative Study of Adsorbents. J. Braz. Chem. Soc., v. 22, nº 3, 558-563, 2011.

GOMES, M. C. S.; PEREIRA, N. C.; AROOYO, P. A. Biodiesel production from degummed soybean oil and glycerol removal using ceramic membrane. Journal of Membrane Science, v. 378, p. 453 - 461, 2011.

HARTMAN, L., LAGO, R.C.A. Rapid Preparation of Fatty Acids Methyl Esters. Laboratory Practice, London, v.22, p.475-476, 1973.

LAGERGREN, S. (1898), Zur theorie der sogenannten adsorption gelöster stoffe, Kungliga Svenska Vetenskapsakademiens. Handlingar, 24 (4) : 1.39.

McCABE, W. L.; SMITH, J. C.; HARRIOTT, P. Unit operations of chemical engineering. 5th ed., New York, McGraw-Hill, Inc. 1993.

MITTELBACH, M.; Diesel fuel derived from vegetable oils, VI; Soecifications and quality control of biodiesel Bioresorse Technology 1996, 56, 7.

MURUGESAN, A.; UMARANI, C.; CHINNUSAMY, T. R.; KRISHNAN, M.; SUBRAMANIAM, R.; NEDUZCHEZHAIN, N. Production and analysis of biodiesel from non-edible oils - A review. Renewable and Sustainable Energy Reviews, v. 13, p. 825-834, 2009.

PEEREBOON, L.; KOENIGSKNCHT, B.; HUNTER, M.;JACKSON, J. E.;MILLER, D. J. Aqueous-phase adsorption of glycerol and propylene glycol onto activated carbon. Carbon, v 45, p. 579-586, 2006.

VASQUES. E. K., Adsorção de Glicerol, Mono e Di-glicerídeos Presentes no Biodiesel Produzido a Partir do Óleo de Soja. UFPR 2010. Dissertação (Mestrado em Tecnologia de Alimentos) - Universidade Federal do Paraná. 2010.

WEBER, W. J.; SMITH, E. H. Activated Carbon: The State of the Art. Environmental Science, v. 29, p. 455-492, 1986. 